University of Wollongong

Research Online

Faculty of Informatics - Papers (Archive)

Faculty of Engineering and Information

Sciences

27-6-2004

\title{
Chain-based extraction of line segments to describe images
}

\author{
A. Chalechale \\ University of Wollongong \\ G. Naghdy \\ University of Wollongong, golshah@uow.edu.au \\ Prashan Premaratne \\ University of Wollongong, prashan@uow.edu.au \\ H. Moghaddasi \\ National Iranian Oil Company, Tehran
}

Follow this and additional works at: https://ro.uow.edu.au/infopapers

Part of the Physical Sciences and Mathematics Commons

\section{Recommended Citation}

Chalechale, A.; Naghdy, G.; Premaratne, Prashan; and Moghaddasi, H.: Chain-based extraction of line segments to describe images 2004.

https://ro.uow.edu.au/infopapers/53

Research Online is the open access institutional repository for the University of Wollongong. For further information contact the UOW Library: research-pubs@uow.edu.au 


\title{
Chain-based extraction of line segments to describe images
}

\author{
Abstract \\ This work presents a novel fast method for line segment extraction, based on a chain code representation \\ of edge maps. It has a parallel nature and can be employed on parallel machines. In the first phase it \\ breaks the macro chains into several micro chains after applying shifting, smoothing and differentiating. \\ The micro chains are then approximated by straight line segments. In the second phase, based on the \\ length and the error criteria, the line segments are grouped into much longer lines. The experimental \\ results show a significant improvement in the number of line segments extracted while their accumulative \\ length keeps high. \\ Disciplines \\ Physical Sciences and Mathematics

\section{Publication Details} \\ This article was originally published as: Chalechale, A, Naghdy, G, Pramaratne, P \& Moghaddasi, H, Chain- \\ based extraction of line segments to describe images, 2004 IEEE International Conference on Multimedia \\ and Expo(ICME '04), 27-30 June 2004, 1, 355-358. Copyright IEEE 2004.
}




\title{
Chain-Based Extraction of Line Segments to Describe Images
}

\author{
Abdolah Chalechale ${ }^{1}$, Golshah Naghdy ${ }^{1}$, Prashan Premaratne ${ }^{1}$, and Hasan Moghaddasi ${ }^{2}$ \\ ${ }^{1}$ School of Electrical, Computer \& Telecommunications Engineering \\ University of Wollongong, Australia \\ $\{$ ac82, golshah, prashan $\} @$ uow.edu.au \\ ${ }^{2}$ National Iranian Oil Company \\ Tehran, Iran \\ moghaddasi@nioc.org
}

\begin{abstract}
The paper presents a novel fast method for line segment extraction based on chain code representation of edge maps. It has a parallel nature and can be employed on parallel machines. In the first phase it breaks the macro chains into several micro chains after applying shifting, smoothing and differentiating. The micro chains are then approximated by straight line segments. In the second phase, based on the length and the error criteria, the line segments are grouped into much longer lines. The experimental results show a significant improvement in the number of line segments extracted while their accumulative length keeps high.
\end{abstract}

\section{Introduction}

Image analysis is one of the most important issues in multimedia processing. There are several low-level image primitives used in computer vision and scene investigation. One of the most serviceable primitives is elementary line segments (ELSs). A line segment, as a primitive geometric object, is frequently used in higher-level processes such as object recognition, stereo matching, target tracking, image registration, and face recognition [i]. Different characteristics of line segments distribution can serve as useful features in image retrieval applications.

An edge map is generally created in the initial stages in most image understanding systems. Although the edge points could be connected on the basis of image connectivity, proximity and orientation, such a representation has limited use since it lacks the compact analytical description of image features required for any vision related tasks. The desired description must also overcome the problems of noise and scatter in the position of edge pixels [2].

Employing lineal features in an image instead of using edge points solely, is noted widely in the literature. Hough transform has been used frequently for line detection [3]. The main concept in this approach is based on voting, where local edges vote for all possible lines they might belong to. The advantage of this approach is that it enables us to detect collinear edge points even when they are all isolated. The method is useful in noisy images when local information around edge pixels is unreliable or unavailable. However, there are several problems with this approach. It is memory hungry and computationally expensive. It also suffers from the so-called connectivity problem, where illusionary lines composed of accidentally collinear edge pixels are falsely detected $[4,5]$.

Burns et al. [6] introduced an approach for the extraction of straight lines in intensity images. It begins at the level of lines directly without going through the intermediate stage of detecting local edges. It utilizes the gradient direction to partition the image into a set of support regions. A leastsquares fitting procedure is then used to fit a line segment to each region. They suggest that this overcomes the difficulties encountered in aggregation when using local operators.

A recursive growing algorithm applied to the edges to identify the line segments in the images is proposed in [7]. Assfalg et al. [8] applied this method for semantic annotation of sports videos. In another approach proposed by Etemadi [2], chains of edge points are obtained using the Marr-Hildreth edge finder. The chains are then segmented into pieces that are symmetric about their centroid. Subsequently, they are related into longer segments. The method is used in [9] for feature matching, which addresses the problem of matching line segments in two images from a sequence of images.

A face feature representation, Line Edge Map (LEM), is proposed in [1] to integrate the structural information with spatial information of a face image by grouping pixels of face edge map to line segments. The polygonal line fitting procedure introduced by Leung and Yang [10] is applied to generate the LEM of a face. The approach in [10] is based on a dynamic strip for fitting a curve with lines. It fits each data point on a curve with two, left-hand and right-hand, strips. The algorithm is run several times with different minimum strip widths.

There are other works which can be categorized as "Line Segment Grouping" approaches $[4,11,12]$. In general, ELSs are obtained by linking edge pixels and approximating to piecewise straight line segments. In particular, in [4] a new method for extracting line segments from edge images is proposed. The method is basically a line segment grouping approach which employs the concept of "line detection by voting". The method uses ELSs as input and groups them as lines with the longest possible length. The algorithm is repeatedly used to hook the remaining un-grouped ELSs into much longer lines.

This paper proposes a new fast method for line segment extraction from edge maps. It has a parallel nature and can be used on parallel machines easily. The method uses the chain codes in the edge map, namely macro chains, for line segment detection. In the first phase; it breaks the macro 
chains into several micro chains by employing the extreme points of the first derivative of shifted-smoothed chain code function. The resulting micro chains are then approximated by straight line segments. In the second phase, the line segments are grouped together based on their proximity (collinearity and nearness). The final set could be tailored for any minimum length and minimum error desired.

The rest of the paper is organized as follows. In the next section the proposed method is described in detail. Section 3 gives the results of experiments, where the performance of the method is compared with three other well-known methods. Conclusions and further works are given in Section 4.

\section{Chain-Based Line Segment Extraction (CBLSE)}

In this section the details of Chain-Based Line Segment Extraction (CBLSE) method are discussed. The input of the method is an edge map $I$ derived by any edge extraction method (e.g. Canny operator) or any thinned sketched image. The method consists of two main phases. In the first phase, by rastering $I$, the start point of an 8-connectivity chain code (see [13]) is followed. The macro chain $A_{i}=$ $\left\{a_{1}, a_{2} \ldots a_{n}\right\}, i=1,2, \ldots, m$; where $m$ is the number of chains in $I$ and $n$ is the chain length; is obtained and put in a chain set $\left\{A_{i}\right\}$. For each $A_{i}$, we then apply the following steps (see Fig. 1):

1. Eliminating Chain Noise: Noisy points which make the chain over oscillating is eliminated by median filtering (such as first peak in Fig. 1-b). Applying a third order one-dimensional median filter on the vector $A_{i}$ reduces the effect of such points adequately.

2. Shifting Operation: The standard chain code representation has the wraparound drawback. For example, a line along $-22.5^{\circ}$ direction is coded as $\{707070 \ldots\}$ (standard chain code representation). To eliminate or reduce such wraparound, we produce a new modified code $B_{i}=\left\{b_{1}, b_{2} \ldots b_{n}\right\}$ for each $A_{i}=\left\{a_{1}, a_{2} \ldots a_{n}\right\}$ by a shifting operation [14] defined recursively as:

$$
\left\{\begin{aligned}
b_{1}= & a_{1} \\
b_{i}= & q_{i}, q_{i} \text { is an integer such that }\left(q_{i}-a_{i}\right) \bmod 8=0 \\
& \text { and }\left|q_{i}-b_{i-1}\right| \text { is minimized for } i=2,3, \ldots n .
\end{aligned}\right.
$$

The line along $-22.5^{\circ}$ direction is now coded as $\{787878 \ldots\}$. Comparison of Fig. 1-b and 1-c shows the wraparound effect.

3. Smoothing Operation: The shifted chain code $B_{i}$ is then smoothed by a five-point Gaussian filter $\{0.1,0.2,0.4,0.2,0.1\}[14] . \Gamma(\theta)$ is the resulted shifted and smoothed waveform where $\theta$ is the traversing variable (Fig. 1-d).

4. First Derivative and Break Extraction: $d \Gamma / d \theta$ determines the rate of change of $\Gamma(\theta)$ with respect to $\theta$. The extreme points of this derivative is considered as break points $\left(\zeta_{i}\right)$, if they are greater than a threshold $\tau$. Because the shifted-smoothed function $d \Gamma / d \theta$ mostly has adjacent values with the difference of 0 or 1 and the maximum of the Gaussian filter used is $0.4,0.3$ is depicted for the threshold value $\tau$ (Fig. 1-e).

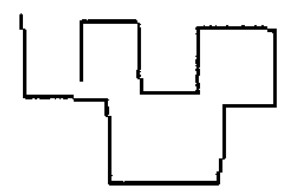

(a)

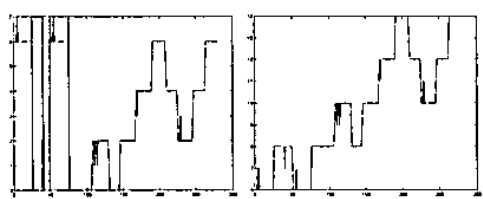

(b)

(c)

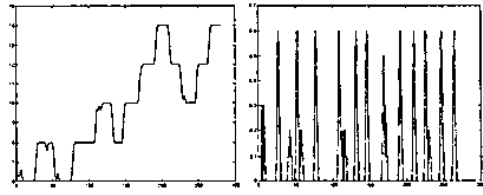

(d)

(e)

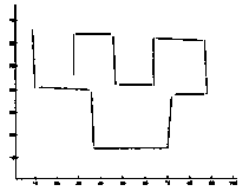

(f)

Figure 1: (a) an example contour, (b) chain code, (c) shifted chain code, (d) smoothed representation, (e) derivative and (f) extracted line segments

The horizontal axis of Figs. 1-b to 1-e is for traversing variable $\theta$ and the vertical axis is the corresponding code value. The axes of Fig. 1-f show the length in terms of pixels.

The line segment $l_{i}$ which connects $\zeta_{i}$ to $\zeta_{i+1}$ is considered as the lineal approximation of the micro chain lays between the two points (Fig. 1-f).

A polygonal approximation for the micro chain from $\zeta_{i}$ to $\zeta_{i+1}$ can also be employed to obtain more line segments. A more accurate representation will be achieved based on chain splitting that subdivides a micro chain successively into two parts until a given criterion is satisfied. However, the experimental results on the test data (Section 3) show that there is no significant improvement in number of line segments and their cumulative length by applying such polygonal approximation. Therefore, the set $\left\{l_{i}\right\}$, where $l_{i}$ is the straight line segment connecting $\zeta_{i}$ to $\zeta_{i+1}$ will be used in the second phase. In the case of using polygonal approximation, $\left\{l_{i}\right\}$ contains all line segments obtained by splitting process.

In the second phase of the method, grouping the line segments obtained in the first phase will produce the final line segments set $\left\{f_{i}\right\}$. The main function of this phase is to replace two short line segments by a single longer one. The decision is based on collinearity and nearness of the line segments. ([12] proposes a metric for this kind of replace- 

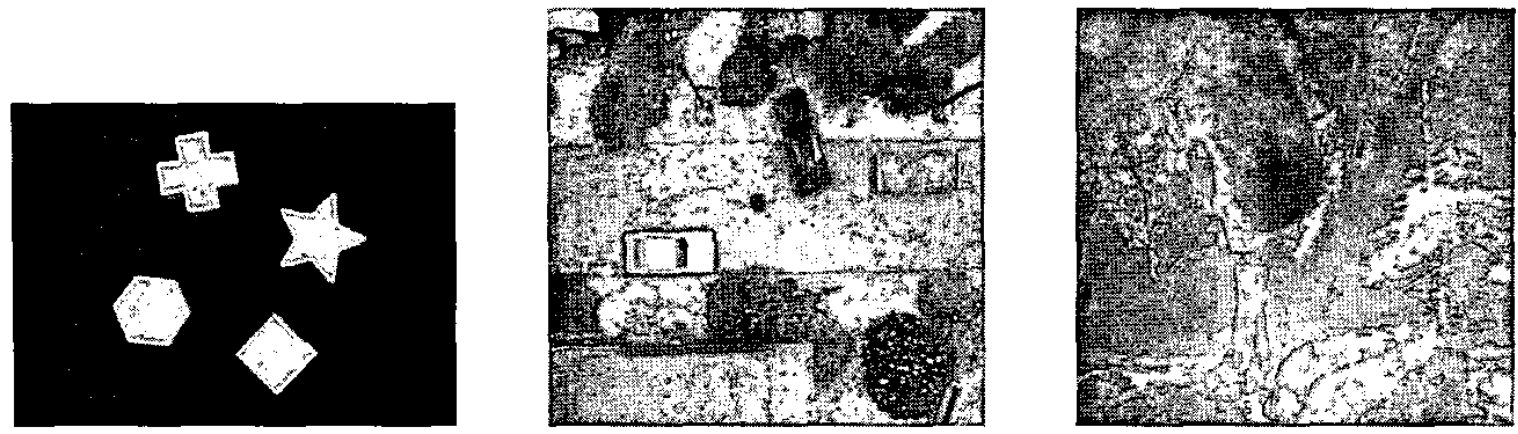

Figure 2: Image \#1 (left), Image \#2 (middle) and Image \#3
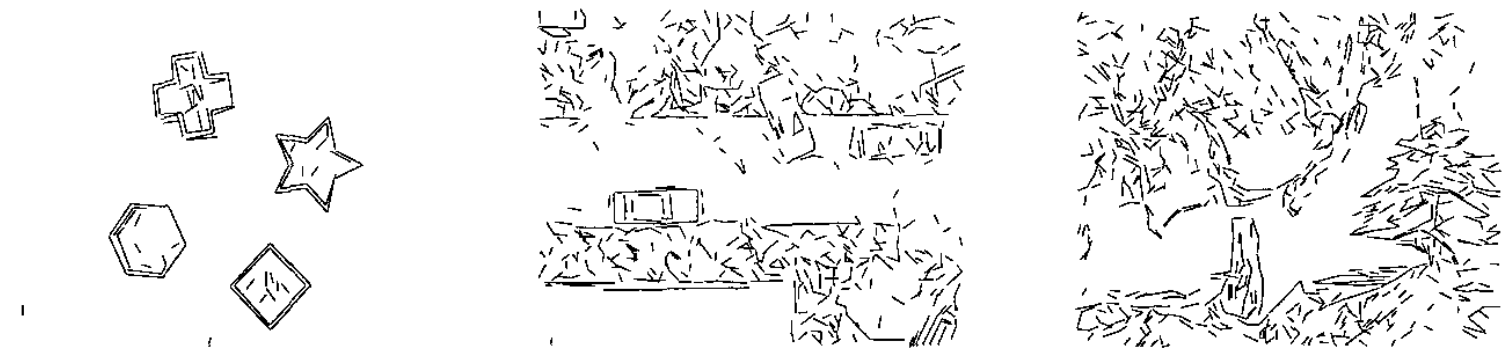

Figure 3: Results of Image \#1 (left), Image \#2 (middle) and Image \#3 using CBLSE method

ment).

The procedure of the second phase is as follows. Suppose we desire to have line segments with minimum length $\lambda$. We first partition $\left\{l_{i}\right\}$ to $\left\{g_{i}\right\}$ and $\left\{k_{i}\right\}$ where $\left\{g_{i}\right\}$ contains the line segments with length greater than or equal to $\lambda$ and $\left\{k_{i}\right\}$ contains the line segments whose length is less than $\lambda$. Then, the proximity of each $k_{i}$ to the rest of $\left\{k_{i}\right\}$ in the direction and the neighborhood is examined. If two different line segments $\left(k_{i}\right.$ and $\left.k_{j}\right)$ are located close to each other and the absolute difference of their directions is less than a predefined value, a line segment that connects the farthest end points is made (namely $n_{i}$ ). The maximum distance (error) of the two line segments from the new one should not exceed an error threshold value $e$. We empirically realized that $e=5$ pixels is a good choice in this study (the space limitation preclude us to explain the details). If the length of the new line segment is greater than or equal to $\lambda$ and the error is less than $e$, then it is kept in a set $\left\{n_{i}\right\}$. Finally, the $\left\{f_{i}\right\}$ is obtained by:

$$
\left\{f_{i}\right\}=\left\{g_{i}\right\} \cup\left\{n_{i}\right\}
$$

\section{Experimental Results}

In this section the results of the proposed method are provided and compared with those obtained using three other approaches. The input images, "FourObjects" (512x371), "TinyTown" (504x480), and "Tree" (504x480) employed in this experiment are also used in [7] (see Fig. 2). The approaches depicted for comparison are the region of support method of Burns et al. [6], edge linking/segmentation, by Etemadi [2], stick-growing by Nelson [7], and the CBLSE method proposed in this paper.
Table I exhibits the number of line segments and their total length for the above four methods (Method 1 to Method 4 respectively). It can be seen that the CBLSE method (Method 4) resulted in more line segments and more accumulative length for the three images. The number of segments and their total length was computed without polygonal approximation. Only segments longer than 10 pixels were considered in all methods. Fig. 3 shows the output of CBLSE method for the three input images.

Table II shows that embedding extra polygonal approximation for micro chains (as discussed in the Section 2) has little effect on the number of line segments extracted and their accumulative length. The results exhibit that in the case of supplementary polygonal approximation, at most $4.5 \%$ improvement in the number of line segments and $3.6 \%$ improvement in their total length is achievable.

\section{Conclusion}

A new fast method for line segment extraction is introduced. The Chain Based Line Segment Extraction (CBLSE) method utilizes chain code representation of edge points and splits the chains in higher curvature points. Multiple chains are extracted from the edge map of the image. In the first phase of the method, each chain is divided into several micro chains by a sequence of shifting, smoothing and differentiating procedures. The straight line segments, connecting the end points of micro chains, are resulted at the end of this stage. In the second phase, small line segments are grouped to make longer lines if the length and the error criteria are satisfied. Experimental results show a significant improvement in the number of line segments extracted and also in their cumulative length. The CBLSE method is com- 
Table I: Number of segments and total length statistics

\begin{tabular}{|c||c|c||c|c||c|c||c|c|}
\hline \multicolumn{1}{|c||}{} & \multicolumn{2}{c||}{ Method 1 } & \multicolumn{2}{c|}{ Method 2 } & \multicolumn{2}{c|}{ Method 3 } & \multicolumn{2}{c|}{ Method 4 } \\
\cline { 2 - 9 } Image\# & Segment & Length & Segment & Length & Segment & Length & Segment & Length \\
\hline \hline \multirow{2}{*}{1} & 50 & 1642 & 58 & 1406 & 61 & 2053 & 96 & 2615 \\
2 & 124 & 2741 & 166 & 2994 & 382 & 8701 & 588 & 9115 \\
3 & 120 & 2053 & 270 & 3904 & 508 & 11354 & 773 & 11698 \\
\hline
\end{tabular}

Table II: The effect of polygonal approximation

\begin{tabular}{|c||c|c||c|c|}
\hline \multicolumn{1}{|c||}{ Image\# } & \multicolumn{4}{c|}{ Method 4 with polygonal approximation } \\
\cline { 2 - 5 } & Segment & Length & Segment Imprv. \% & Length Imprv. \% \\
\hline \hline 1 & 99 & 2646 & 3.1 & 1.2 \\
2 & 598 & 9243 & 1.7 & 1.4 \\
3 & 808 & 12120 & 4.5 & 3.6 \\
\hline
\end{tabular}

pared with three other well-known methods in the literature and shows $52 \%$ improvement in the number of line segment while the accumulative length is always higher than the others. Polygonal approximation has negligible effect on the overall performance.

As each chain is processed individually in the first stage, the method can be employed on parallel machines. We intend to apply the CBLSE method for content based image retrieval where the input example is a hand-drawn sketch (sketch based image retrieval).

Acknowledgments. The authors would like to acknowledge Dr. Randal C. Nelson, University of Rochester, NY, USA, for his help in providing the test data used in this work. The first author is financially supported by the Ministry of Science, Research and Technology of I.R.Iran.

\section{References}

[1] Y. Gao and M. K. H. Leung, "Face recognition using line edge map," IEEE Transactions on Pattern Analysis and Machine Intelligence, vol. 24, no. 6, pp. 764$779,2002$.

[2] A. Etemadi, "Robust segmentation of edge data," in IEE Intermational Conference on Image Processing and its Applications., 1992, pp. 311-314.

[3] R. O. Duda and P. E. Hart, "Use of the Hough transformation to detect lines and curves in pictures," Communications of the $A C M$, vol. 15 , no. $12, \mathrm{pp} .11-15$, 1972.

[4] J.-S. Jang and K.-S. Hong, "Fast line segment grouping method for finding globally more favorable line segments," Pattern Recognition, vol. 35, no. 10, pp. 2235-2247, 2002.

[5] J. Zhu and Y. Zhao, "Vehicle license image segmentation using wavelet transform," in Proceedings of IEEE International Symposium on Intelligent Multimedia, Video and Speech Processing., 2001, pp. 26770 .
[6] J. B. Burns, A. R. Hanson, and E. M. Riseman, "Extracting straight lines," IEEE Trans. on Pattern Analysis and Machine Intelligence, vol. 18, no. 4, pp. 425$455,1986$.

[7] R. C. Nelson, "Finding line segments by stick growing," IEEE Transactions on Pattern Analysis and Machine Intelligence, vol. 16, no. 5, pp. 519-523, 1994.

[8] J. Assfalg, M. Bertini, C. Colombo, and A. D. Bimbo, "Semantic annotation of sports videos," IEEE Multimedia, vol. 9, no. 2, pp. 52-60, 2002.

[9] Y. L. Chang and J. K. Aggarwal, "Line correspondence from cooperating spatial and temporal grouping processes for a sequence of images," Computer Vision and Image Understanding, vol. 67, no. 2, pp. 186-201, 1997.

[10] M. K. Leung and YH Yang, "Dynamic two-strip algorithm in curve fitting," Pattern Recognition, vol. 23, no. 1-2, pp. 69-79, 1990.

[11] M. Boldt, R. Weiss, and E. Riseman, "Token-based extraction of straight lines," IEEE Transactions on Systems, Man and Cybernetics, vol. 19, no. 6, pp. 1581$1594,1989$.

[12] P. F. M. Nacken, "A metric for line segments," IEEE Transactions on Pattern Analysis and Machine Intelligence, vol. 15, no. 12, pp. 1312-1318, 1993.

[13] R. C. Gonzalez and R. E. Woods, Digital Image Processing, Addison-Wesley, 1992.

[14] H. Li, B. S. Manjunath, and S. K. Mitra, "A contourbased approach to multisensor image registration," IEEE Transactions on Image Processing, vol. 4, no. 3, pp. 320-334, 1995. 